\title{
Surfactant Penetration into Human Skin from Sodium Dodecyl Sulfate and Lauramidopropyl Betaine Mixtures
}

\author{
Stephanie A.V. Morris, ${ }^{1,2}$ Lijing Xu, ${ }^{1}$ K.P. Ananthapadmanabhan, ${ }^{1}$ and Gerald B. Kasting ${ }^{1 *}$ \\ ${ }^{1}$ James L. Winkle College of Pharmacy, University of Cincinnati, Cincinnati, Ohio 45267-0514, USA; \\ xulj@mail.uc.edu, ananthky@ucmail.uc.edu, Gerald.Kasting@uc.edu \\ ${ }^{2}$ Present address: L’Oreal Research and Innovation Dept., Clark, NJ; Stephanie.Morris@rd.loreal.com

\section{SUPPORTING INFORMATION}

Correspondence:

Gerald B. Kasting

James L. Winkle College of Pharmacy

University of Cincinnati Academic Health Center

P.O. Box 670514

Cincinnati, Ohio 45267-0514

Phone: (513) 558-1817

Fax: (513) 558-3233

Email: Gerald.Kasting@uc.edu 


\section{Regular solution calculations}

We choose here the nomenclature employed by Holmberg et al. ${ }^{1}$ and confine the analysis to binary surfactant systems, which become ternary systems when dissolved in water. The extension to multicomponent systems is nicely described by Holland and Rubingh. ${ }^{2}$ More elaborate models including the effect of counterions ${ }^{3}$ and molecular thermodynamics ${ }^{4}$ are available, but are not necessary to describe the data obtained in this study.

The chemical potential of a surfactant species $i$ in solution, $\mu_{\mathrm{i}}\left(\mathrm{J} \mathrm{mol}^{-1}\right)$, can be written as $\mathrm{s}^{1-2,5}$

$$
\mu_{i}=\mu_{i}^{0}+R T \ln c_{i}
$$

where $\mu_{\mathrm{i}}^{0}$ is the standard chemical potential of surfactant $i\left(\mathrm{~J} \mathrm{~mol}^{-1}\right), R$ is the gas constant $\left(\mathrm{J} \mathrm{mol}^{-1} \mathrm{~K}^{-1}\right), T$ is temperature $(\mathrm{K})$, and $c_{\mathrm{i}}$ is the concentration of surfactant $i$ in the bulk solution $\left(\mathrm{mol} \mathrm{L}^{-1}\right)$. In a nonideal mixture, the chemical potential of surfactant $i$ in a mixed micelle, $\mu_{\mathrm{i}}^{\mathrm{m}}\left(\mathrm{J} \mathrm{mol}^{-1}\right)$ can be calculated as

$$
\mu_{i}^{m}=\mu_{i}^{0 m}+R T \ln f_{i}^{m} x_{i}^{m}
$$

where $\mu_{\mathrm{i}}^{0 \mathrm{~m}}$ is the chemical potential of surfactant $i$ in the pure micelle $\left(\mathrm{J} \mathrm{mol}{ }^{-1}\right), f_{\mathrm{i}}^{\mathrm{m}}$ is the activity coefficient of surfactant $i$ in the mixed micelle, and $x_{i}^{m}$ is the mole fraction of surfactant $i$ in the mixed micelle. If the activity coefficient of free monomers is equal to unity, then

$$
\mu_{i}^{n}=\mu_{i}^{0 n}+R T \ln c_{i}^{n}
$$

where $\mu_{\mathrm{i}}^{\mathrm{n}}$ is the chemical potential of monomeric surfactant $i\left(\mathrm{~J} \mathrm{~mol}^{-1}\right), \mu_{\mathrm{i}}{ }^{0 \mathrm{n}}$ is the standard chemical potential of monomeric surfactant $i\left(\mathrm{~J} \mathrm{~mol}^{-1}\right)$, and $c_{\mathrm{i}}^{\mathrm{n}}$ is the concentration of monomeric surfactant $i$ (mol

$\mathrm{L}^{-1}$ ). Note that eq S2 employs a rational activity model, whereas eq S3 a practical activity model with millimolar concentrations; consequently the numerical values of $\mu_{01}^{\mathrm{m}}$ and $\mu_{01}^{\mathrm{n}}$ are quite different. This choice is a consequence of the widely different concentration regimes in which micellar and monomeric surfactant exist. ${ }^{6}$ Using a phase separation model for micellization, ${ }^{2}$ 


$$
\mu_{i}^{0 m}=\mu_{i}^{0 n}+R T \operatorname{lnCMC}{ }_{i}
$$

where $\mathrm{CMC}_{\mathrm{i}}$ is the critical micelle concentration $(\mathrm{CMC})$ of pure surfactant $i\left(\mathrm{~mol} \mathrm{~L}^{-1}\right)$. At equilibrium, $\mu_{\mathrm{i}}^{\mathrm{m}}=$ $\mu_{\mathrm{i}}^{\mathrm{n}}$. Therefore, from eqs S2-S4, ${ }^{5}$

$$
c_{i}^{n}=f_{i}^{m} x_{i}^{m} \mathrm{CMC}_{i} .
$$

Thus, in a mixture of surfactant species " 1 " and "2" in a binary surfactant solution above the CMC,

$$
\begin{aligned}
& c_{1}^{n}=f_{1}^{m} x_{1}^{m} \mathrm{CMC}_{1} \\
& c_{2}^{n}=f_{2}^{m}\left(1-x_{1}^{m}\right) \mathrm{CMC}_{2} .
\end{aligned}
$$

Below the CMC,

$$
\begin{aligned}
& c_{1}^{n}=x_{1} c_{\mathrm{tot}} \\
& c_{2}^{n}=\left(1-x_{1}\right) c_{\mathrm{tot}}
\end{aligned}
$$

where $x_{1}$ is the mole fraction of surfactant 1 in the bulk solution and $c_{\text {tot }}$ is the total surfactant concentration $\left(\mathrm{mol} \mathrm{L}^{-1}\right)$.

\section{Calculation of $\beta$ parameter and mixed CMCs}

Eliminating $c_{1}^{\mathrm{n}}$ from eqs $\mathrm{S} 6$ and $\mathrm{S} 8$ and substituting $\mathrm{CMC}_{\text {mix }}$ for $c_{\text {tot }}$ yields

$$
x_{1}^{m}=\frac{x_{1} \mathrm{CMC}_{\mathrm{mix}}}{f_{1}^{m} \mathrm{CMC}_{1}}
$$

where $\mathrm{CMC}_{\text {mix }}$ is the critical micelle concentration of the surfactant mixture ( $\mathrm{mol} \mathrm{L}^{-1}$ ) at a given solution composition. Similarly, eliminating $c_{2}^{n}$ from eqs $\mathrm{S} 7$ and $\mathrm{S} 9$ and substituting $\mathrm{CMC}_{\text {mix }}$ for $c_{\text {tot }}$ yields

$$
\left(1-x_{1}^{m}\right)=\frac{\left(1-x_{1}\right) \mathrm{CMC}_{\mathrm{mix}}}{f_{2}^{m} \mathrm{CMC}_{2}}
$$


Eliminating $x_{1}{ }^{m}$ from equations $\mathrm{S} 10$ and $\mathrm{S} 11$ yields

$$
\frac{1}{\mathrm{CMC}_{\mathrm{mix}}}=\frac{x_{1}}{f_{1}^{m} \mathrm{CMC}_{1}}+\frac{1-x_{1}}{f_{2}^{m} \mathrm{CMC}_{2}}
$$

Activity coefficients of surfactants 1 and 2 in the mixed micelle, $f_{1}{ }^{\mathrm{m}}$ and $f_{2}{ }^{\mathrm{m}}$, are defined using the regular solution theory: ${ }^{1}$

$$
\begin{aligned}
& \ln f_{1}^{m}=\left(x_{2}^{m}\right)^{2} \beta \\
& \ln f_{2}^{m}=\left(x_{1}^{m}\right)^{2} \beta
\end{aligned}
$$

where $\beta$ is the interaction parameter between Species 1 and 2 .

At this point one has two choices as to how to calculate the interaction parameter $\beta$. The choice is determined, in part, by the amount of data available. The options are nicely described by Sarmoria et al. ${ }^{7}$, who refer to them as $\beta^{\text {avg }}$ and $\beta^{\text {opt }}$. We describe both here, but have chosen to use the $\beta^{\text {opt }}$ method for the reasons described by Sarmoria et al.

$\beta_{\text {avg }}$ determination : Eliminating $f_{1} \mathrm{~m}$ from eqs $\mathrm{S} 10$ and $\mathrm{S} 13$ yields

$$
\beta=\frac{\ln \left[\frac{x_{1} \mathrm{CMC}_{\operatorname{mix}}}{x_{1}^{m} \mathrm{CMC}_{1}}\right]}{\left(1-x_{1}^{m}\right)^{2}}
$$

Similarly, eliminating $f_{2}^{\mathrm{m}}$ from eqs S11 and S14 yields

$$
\beta=\frac{\ln \left[\frac{\left(1-x_{1}\right) \mathrm{CMC}_{\mathrm{mix}}}{\left(1-x_{1}^{m}\right) \mathrm{CMC}_{2}}\right]}{\left(x_{1}^{m}\right)^{2}} .
$$

Eliminating $\beta$ from eqs S15 and S16 yields eq S17.

$$
\frac{\left(x_{1}^{m}\right)^{2} \ln \left[\frac{x_{1} \mathrm{CMC}_{\mathrm{mix}}}{x_{1}^{m} \mathrm{CMC} C_{1}}\right]}{\left(1-x_{1}^{m}\right)^{2} \ln \left[\frac{\left(1-x_{1}\right) \mathrm{CMC}_{\operatorname{mix}}}{\left(1-x_{1}^{m}\right) \mathrm{CMC}_{2}}\right]}=1
$$


Microsoft Excel Solver ${ }^{\mathrm{TM}}$ is used to solve equation $\mathrm{S} 17$ for $\mathrm{x}_{1}{ }^{\mathrm{m}}$. This method yields a $\beta$ parameter value at each given solution composition. The arithmetic average of these values is defined as $\beta^{\text {avg }}$.

$\beta^{\text {opt }}$ determination: The objective is to minimize the squared residuals of predicted $\mathrm{CMC}_{\text {mix }}$ from observed $\mathrm{CMC}_{\text {mix }}$ for all available mixtures. The sum of squared residuals (SSR) is defined as:

$$
\mathrm{SSR}=\sum_{i}\left(\mathrm{CMC}_{\mathrm{mix}}^{\mathrm{pred}}-\mathrm{CMC}_{\mathrm{mix}}^{\mathrm{obs}}\right)^{2}
$$

where $\mathrm{CMC}_{\text {mix }}^{\text {pred }}$ is calculated from eq. $\mathrm{S} 12$ and $\mathrm{CMC}_{\text {mix }}^{\text {obs }}$.represents experimental data. A suitable iteration scheme is as follows:

\section{Outer loop}

1. Make an estimate of $\beta$. For the initial estimate, use $\beta=0$ or draw from experience, e.g. chose $\beta^{\text {avg }}$ (eqs. S15-17).

2. Make estimates $x_{1}^{m}$ for each experimental composition. For $\beta=0$, choose $x_{1}^{m}=x_{1}$. For $\beta=\beta^{\text {avg }}$, choose the $x_{1}^{m}$ resulting from eq. S17.

Inner loop

3. Calculate $f_{1}^{m}$ and $f_{2}^{m}$ for each composition from eqs. S13 and S14.

4. Revise the $x_{1}^{m}$ according to eq. S19, which arises from Holland and Rubingh's ${ }^{2}$ eq. 13 in the case of a binary solution.

$$
x_{1}^{m}=\frac{x_{1} f_{2}^{m} \mathrm{CMC}_{2}}{x_{1} f_{2}^{m} \mathrm{CMC}_{2}+\left(1-x_{1}\right) f_{1}^{m} \mathrm{CMC}_{1}}
$$

5. Iterate Steps 3 and 4 until the solution converges. We used the GRG nonlinear routine in Microsoft Excel's Solver ${ }^{\mathrm{TM}}$ addin.

Close inner loop

6. Calculate $\mathrm{CMC}_{\mathrm{mix}}^{\mathrm{pred}}$ for each composition from eq. S12, then SSR from eq. S18.

7. Choose another value of $\beta$ so as to optimally reduce SSR, then go to Step 1 . For small problems and moderate accuracy this can be done graphically. To automate, we suggest the secant algorithm. 


\section{Close outer loop}

\section{Calculation of monomer and micelle compositions above the mixed CMC}

These calculations assume the total surfactant concentration $c_{\text {tot }}$, the bulk mole fractions $x_{1}$ and $x_{2}, \mathrm{CMC}_{1}$ and $\mathrm{CMC}_{2}$, and the interaction parameter $\beta$ are all known. It should be emphasized that the micellar mole fractions $x_{i}^{m}$ and activity coefficients $f_{i}^{m}$ are not those at the mixed CMC for $c_{\text {tot }}>\mathrm{CMC}_{\text {mix. }}$. Mass balance considerations require that $x_{i}^{m} \rightarrow x_{i}$ for $c_{\text {tot }} \gg \mathrm{CMC}_{\text {mix. }}$. The equations are written for binary surfactant mixtures, but can be generalized for multicomponent systems. ${ }^{2}$

The concentrations of surfactants 1 and 2 in the mixed micelle, $c_{1}{ }^{\mathrm{m}}$ and $c_{2}{ }^{\mathrm{m}}$, can be calculated using mass balance,

$$
\begin{aligned}
& c_{1}^{m}=x_{1} c_{\text {tot }}-c_{1}^{n} \\
& c_{2}^{m}=\left(1-x_{1}\right) c_{\text {tot }}-c_{2}^{n} .
\end{aligned}
$$

Therefore $x_{1}{ }^{m}$ can be calculated as

$$
x_{1}^{m}=\frac{x_{1} c_{\mathrm{tot}}-c_{1}^{n}}{c_{\mathrm{tot}}-M} .
$$

where

$$
M=c_{1}^{n}+c_{2}^{n}
$$

Rearranging eqs. $S 6$ and $S 22$ yields

$$
x_{1}^{m}=\frac{c_{1}^{n}}{f_{1}^{m} \mathrm{CMC}_{1}}=\frac{x_{1} c_{\mathrm{tot}}-c_{1}^{n}}{c_{\mathrm{tot}}-M} .
$$

Similarly, eqs. S6 and S7 yield

$$
x_{2}^{m}=\frac{c_{2}^{\mathrm{n}}}{f_{2}^{m} \mathrm{CMC}_{2}}=1-\frac{c_{1}^{n}}{f_{1}^{m} \mathrm{CMC}_{1}} .
$$


Eliminating $c_{2}{ }^{n}$ from eqs. S24 and S25 yields a quadratic equation,

$$
c_{1}^{n}\left(c_{\text {tot }}-f_{2}^{m} \mathrm{CMC}_{2}+f_{1}^{m} \mathrm{CMC}_{1}\right)+\left(c_{1}^{n}\right)^{2}\left[\frac{f_{2}^{m} \mathrm{CMC}_{2}}{f_{1}^{m} \mathrm{CMC}_{1}}-1\right]-x_{1} c_{\text {tot }} f_{1}^{m} \mathrm{CMC}_{1}=0 .
$$

The solution to eq. S26 is

$$
x_{1}^{m}=\frac{c_{1}^{n}}{f_{1}^{m} \mathrm{CMC}_{1}}=\frac{-\left(\mathrm{c}_{\mathrm{tot}}-\varphi\right)+\left[\left(c_{\mathrm{tot}}-\varphi\right)^{2}+4 x_{1} \mathrm{c}_{\mathrm{tot}} \varphi\right]^{1 / 2}}{2 \varphi} .
$$

where

$$
\varphi=f_{2}^{m} \mathrm{CMC}_{2}-f_{1}^{m} \mathrm{CMC}_{1}
$$

A more general relationship for $x_{i}^{m}$, extendable to multicomponent systems, is given by Holland and Rubingh $^{2}$ (their eq. 10):

$$
x_{i}^{m}=\frac{x_{i} c_{\text {tot }}}{c_{\text {tot }}+f_{i}^{m} \mathrm{CMC}_{i}-M}
$$

A suitable procedure for solving these relationships for a given bulk solution composition is as follows:

1. Make initial estimates for $x_{1}^{m}$ and $x_{2}^{m}=1-x_{1}^{m}$ from prior experience.

2. Calculate $f_{1}^{m}$ and $f_{2}^{m}$ from eqs. S13 and S14.

3. Calculate $c_{1}^{n}$ and $c_{2}^{n}$ from eqs. $\mathrm{S} 6$ and $\mathrm{S7}$, then calculate $M$ from eq. $\mathrm{S} 23$.

4. Calculate revised $x_{1}^{m}$ and $x_{2}^{m}$ from eq. $S 29, i=1,2$.

5. Repeat Steps 2-4 until process converges. As in the $\beta$ parameter determination, we used the GRG nonlinear routine in Microsoft Excel Solver ${ }^{\mathrm{TM}}$ to optimize the values of $x_{1}^{m}$.

Surfactant equilibrium distributions and associated parameters in SDS/LAPB $/{ }^{14} \mathrm{C}-\mathrm{SDS}$ mixtures determined using the Holland and Rubingh model are listed in Tables S1 and S2. Mole fraction of each surfactant in the mixed micelle determined using this model as a function of bulk solution composition is 
shown in Figure S1. In both tables "1" denotes SDS $+{ }^{14}$ C-SDS and " 2 " denotes LAPB. Species 1 is subdivided into " $1 a$ " (SDS) and " $1 b$ " $\left({ }^{14} C-S D S\right)$.

Table S1. Equilibrium distributions in SDS/LAPB/ $/{ }^{14} \mathrm{C}$-SDS mixtures at the mixed CMC. Values were determined using Holland and Rubingh's pseudo-phase separation model ${ }^{2}$ with $\beta=-4.3$.

\begin{tabular}{cccccccccc}
\hline$x_{1 \mathrm{a}}$ & $x_{1 \mathrm{~b}}$ & $x_{2}$ & $x_{1}{ }^{\mathrm{m}}$ & $x_{2}{ }^{\mathrm{m}}$ & $f_{1}{ }^{\mathrm{m}}$ & $f_{2}{ }^{\mathrm{m}}$ & $\begin{array}{c}c_{1 \mathrm{a}}{ }^{\mathrm{n}}, \\
\mathrm{mM}\end{array}$ & $\begin{array}{c}c_{1 b^{\mathrm{n}}} \times \\
10^{3}, \\
\mathrm{mM}\end{array}$ & $c_{2}{ }^{\mathrm{n}}, \mathrm{mM}$ \\
\hline 0 & 0.0013 & 0.9987 & 0.013 & 0.987 & 0.015 & 0.999 & 0 & 0.163 & 1.132 \\
0.0999 & 0.0013 & 0.8989 & 0.1965 & 0.803 & 0.062 & 0.847 & 0.044 & 0.560 & 0.862 \\
0.2497 & 0.0013 & 0.7490 & 0.2739 & 0.726 & 0.104 & 0.724 & 0.182 & 0.924 & 0.615 \\
0.4994 & 0.0013 & 0.4994 & 0.3568 & 0.643 & 0.169 & 0.578 & 0.588 & 1.496 & 0.329 \\
0.7490 & 0.0013 & 0.2497 & 0.4427 & 0.557 & 0.263 & 0.430 & 1.371 & 2.327 & 0.124 \\
0.8989 & 0.0013 & 0.0999 & 0.5298 & 0.470 & 0.387 & 0.299 & 2.423 & 3.426 & 0.035 \\
0.9987 & 0.0013 & 0 & 1 & 0 & 1 & - & 6.999 & 7.523 & 0.000 \\
\hline
\end{tabular}

Table S2. Equilibrium distributions in SDS/LAPB/ $/{ }^{14} \mathrm{C}$-SDS mixtures at total surfactant concentration $50.064 \mathrm{mM}$. Values were determined using as in Table S1 with $\beta=-4.3$.

\begin{tabular}{cccccccccc}
\hline$x_{1 \mathrm{a}}$ & $x_{1 \mathrm{~b}}$ & $x_{2}$ & $x_{1}{ }^{\mathrm{m}}$ & $x_{2}{ }^{\mathrm{m}}$ & $f_{1}{ }^{\mathrm{m}}$ & $f_{2}{ }^{\mathrm{m}}$ & $\begin{array}{c}c_{1 \mathrm{a}}^{n} \\
\mathrm{mM}\end{array}$ & $\begin{array}{c}c_{1 \mathrm{~b}}^{n} \times \\
10^{3}, \mathrm{mM}\end{array}$ & $c_{2}^{n}, \mathrm{mM}$ \\
\hline 0 & 0.0013 & 0.9987 & 0.0013 & 0.9987 & 0.014 & 1.000 & 0 & 0.148 & 1.1326 \\
0.0999 & 0.0013 & 0.8989 & 0.1027 & 0.8973 & 0.031 & 0.956 & 0.0223 & 0.284 & 0.9741 \\
0.2497 & 0.0013 & 0.7490 & 0.2518 & 0.7482 & 0.090 & 0.761 & 0.1582 & 0.805 & 0.6467 \\
0.4994 & 0.0013 & 0.4994 & 0.4911 & 0.5089 & 0.328 & 0.354 & 1.1273 & 2.869 & 0.2007 \\
0.7490 & 0.0013 & 0.2497 & 0.7306 & 0.2694 & 0.732 & 0.101 & 3.7403 & 6.347 & 0.0285 \\
0.8989 & 0.0013 & 0.0999 & 0.8869 & 0.1131 & 0.946 & 0.034 & 5.8739 & 8.306 & 0.0038 \\
0.9987 & 0.0013 & 0 & 1 & 0 & 1 & 0 & 6.9983 & 8.907 & 0 \\
\hline
\end{tabular}

1 denotes SDS $+{ }^{14} \mathrm{C}$-SDS as one surfactant species

2 denotes LAPB

a denotes SDS

$b$ denotes ${ }^{14} \mathrm{C}$-SDS

$x_{i}$, mole fraction of surfactant species $i$ in the bulk solution

$x_{i}^{m}($ ref $)$, mole fraction of surfactant species $i$ in the mixed micelle (refined)

$f_{i}^{\mathrm{m}}$, activity coefficient of surfactant species $i$ in the mixed micelle $c_{i}{ }^{n}$, monomeric concentration of surfactant species $i$ 

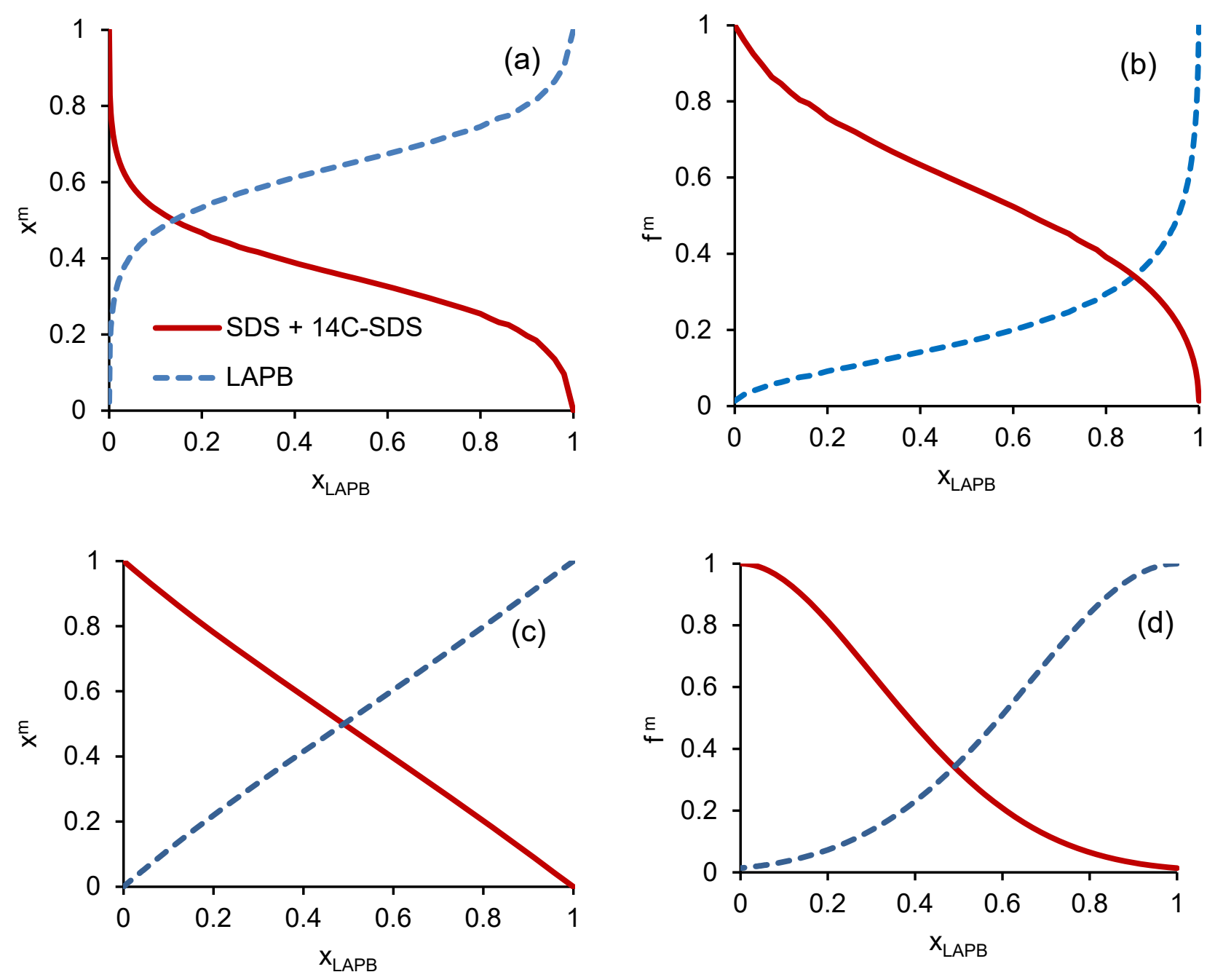

Figure S1. Predicted (a) mole fraction $x^{\mathrm{m}}$ and (b) activity coefficient $f^{\mathrm{m}}$ of either SDS $+{ }^{14} \mathrm{C}-\mathrm{SDS}$ or LAPB in the mixed micelle versus mole fraction of LAPB in the bulk solution ( $x_{\mathrm{LAPB}}$ ) in SDS/LAPB $/{ }^{14} \mathrm{C}-\mathrm{SDS}$ mixtures at the mixed CMC. Panels $(c)$ and $(d)$ show the same information with a constant total surfactant concentration $c_{\text {tot }}=50 \mathrm{mM}$. Values were determined using Holland and Rubingh's pseudo-phase separation model ${ }^{2}$ with $\beta=-4.3$.

The monomer concentrations in mixed surfactant systems at total surfactant concentrations above the mixed $\mathrm{CMC}$, i.e. $c_{\text {tot }}>\mathrm{CMC}_{\text {mix }}$, were determined by a separate iteration procedure. These calculations employed the multicomponent method described by Holland and Rubingh ${ }^{2}$ and were confirmed to be equivalent to Clint's binary mixture calculation. ${ }^{5}$ The procedure included eqs. S13 and S14 and also the following relationships:

$$
c_{1}^{n}=f_{1}^{m} x_{1}^{m} \mathrm{CMC}_{1}
$$




$$
\begin{aligned}
& c_{2}^{n}=f_{2}^{m} x_{2}^{m} \mathrm{CMC}_{2} . \\
& M=c_{1}^{n}+c_{2}^{n} \\
& x_{i}^{m}=\frac{x_{i} c_{\mathrm{tot}}}{c_{\mathrm{tot}}+f_{i}^{m} \mathrm{CMC}_{i}-M} ; i=1,2
\end{aligned}
$$

It should be noted that the values of $f_{i}^{m}$ and $x_{i}^{m}$ emerging from eqs. S30-S33 differ substantially from those calculated from eqs. S12-S19 for the case $c_{\text {tot }} \gg \mathrm{CMC}_{\text {mix }}$, despite sharing the same value of $\beta$. This is a consequence of mass balance. At high surfactant concentrations, the micellar composition necessarily approaches that of the bulk solution.

Concentrations of monomeric SDS $\left(c_{1 \mathrm{a}}^{n}\right)$ and ${ }^{14} \mathrm{C}$-SDS $\left(c_{1 \mathrm{~b}}^{n}\right)$, were then calculated by assuming that SDS and ${ }^{14} \mathrm{C}-\mathrm{SDS}$ partition throughout the mixture equally, giving

$$
c_{1 \mathrm{a}}^{n}=\frac{c_{1 \mathrm{a}}}{c_{1}} c_{1}^{n} ; c_{1 \mathrm{~b}}^{n}=\frac{c_{1 \mathrm{~b}}}{c_{1}} c_{1}^{n}
$$

where $c_{1 \mathrm{a}}$ and $c_{1 \mathrm{~b}}$ represent the bulk solution concentrations and $c_{1}=c_{1 \mathrm{a}}+c_{1 \mathrm{a}}$. 


\section{CMC determinations for SDS and LAPB}

Representative plots of surface tension vs. log concentration of the SDS and LAPB raw materials used in this study are shown in Figure S2. CMCs were estimated as the concentration yielding the minimum surface tension, as indicated on the figure. The regression lines refer to surface excess estimates according to the Gibbs adsorption isotherm (calculations not shown).
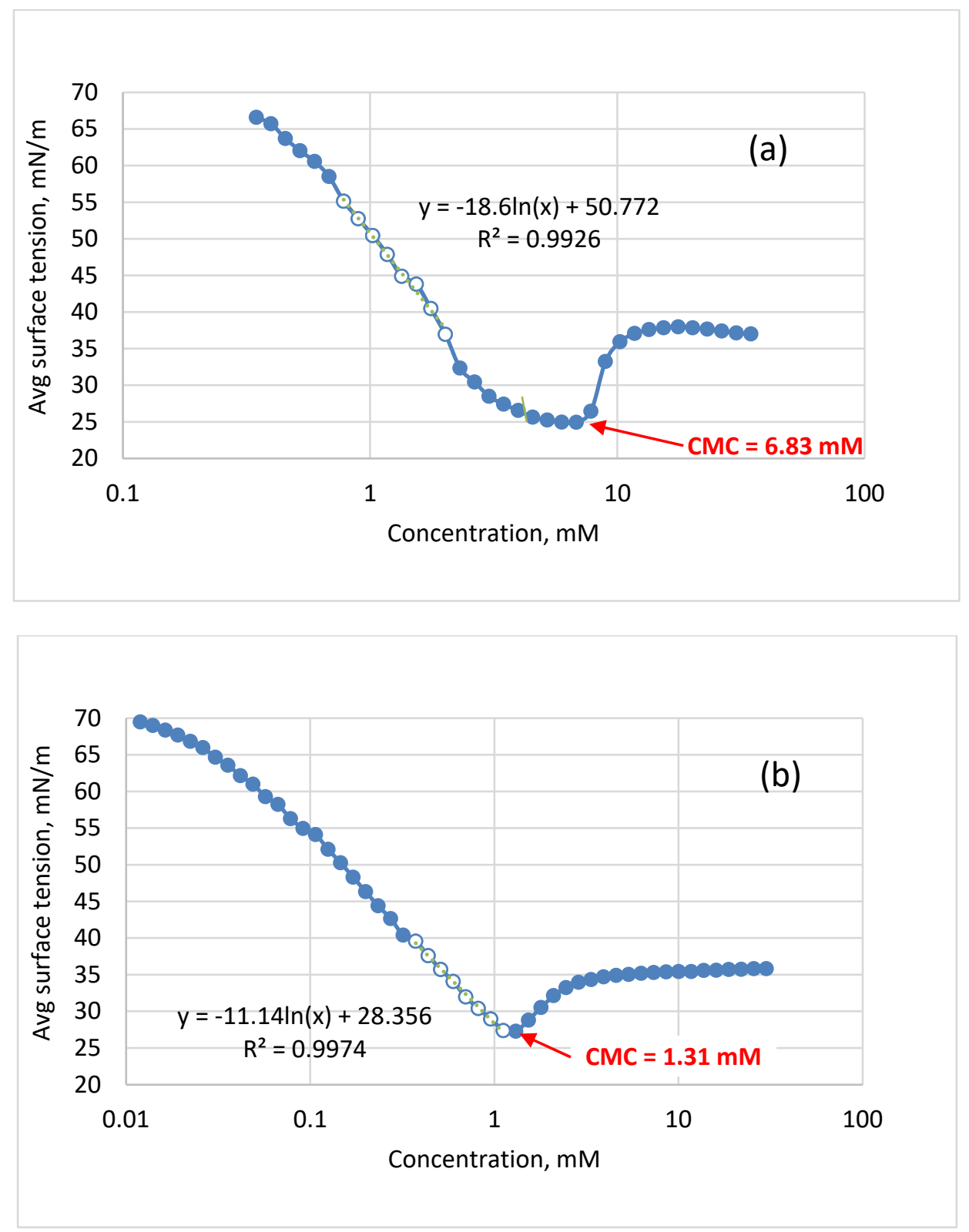

Figure S2. Surface tension versus concentration for dilutions of the raw materials in the present study with DI water at $25^{\circ} \mathrm{C}$. (a) SDS; (b) LAPB. 


\section{Low shear viscosity}

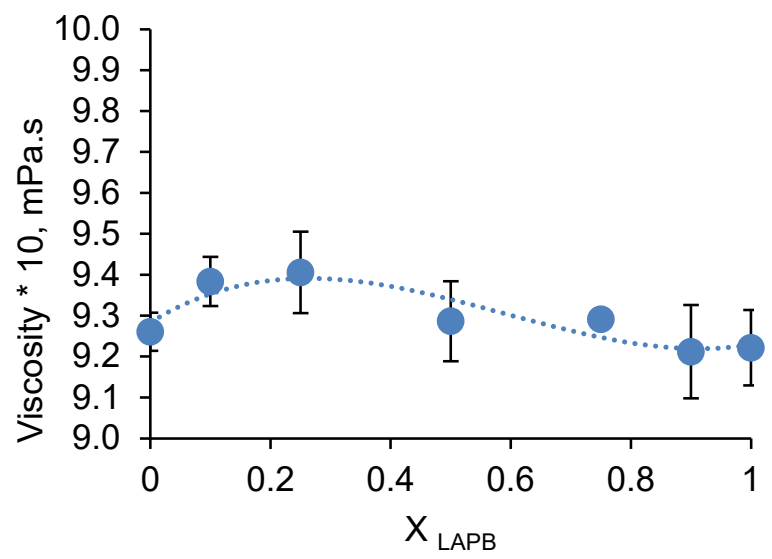

Figure S2. Low shear viscosity and (b) dielectric constant of 30mM SDS/LAPB mixtures versus mole fraction of LAPB in the bulk solution. Viscosities were measured at room temperature using a Ubbelohde viscometer according to the protocol described in ASTM D446-07.

\section{References}

1. Holmberg, K.; Jonsson, B.; Kronberg, B.; Lindman, B., Mixed micelles. In Surfactants and Polymers in Aqueous Solution, Second ed.; John Wiley \& Sons, Ltd.: Chichester, England, 2003; pp 119-138.

2. Holland, P. M.; Rubingh, D. N., Nonideal multicomponent mixed micelle model. J. Phys. Chem. 1983, 87, 1984-1990.

3. Kamrath, R. F.; Franses, E. I., Thermodynamics of mixed micellization. Pseudo-phase separation models. Ind. Eng. Chem. Fundam. 1983, 22, 230-239.

4. Puvvada, S.; Blankschtein, D., Thermodynamic description of micellization, phase behavior and phase separation of aqueous solutions of surfactant mixtures. J. Phys. Chem. 1992, 96, 5567-5579.

5. Clint, J. H., Micellization of mixed nonionic surface active agents. J. Chem. Soc., Faraday Trans. 1 1975, 71, 1327-1334.

6. Evans, D. F.; Wennerstrom, H., The Colloidal Domain: Where Physics, Chemistry, Biology, and Technology Meet. Wiley-VCH: USA, 1999.

7. Sarmoria, C.; Puvvada, S.; Blankschtein, D., Prediction of critical micelle concentrations of binary mixtures of nonideal surfactant mixtures. Langmuir 1992, 8, 2690-2697. 